\title{
COMPONENTES DE CAMBIO Y ECONOMÍA REGIONAL: EL SECTOR MANUFACTURERO EN EL ESTADO DE BAJA CALIFORNIA Y EN EL MUNICIPIO DE MEXICALI (1985-1988)
}

\author{
Por \\ Ma. Dolores Sánchez Soler* \\ Guadalupe Ortega Villa* \\ Edna E. Montoya Sánchez*
}

\begin{abstract}
RESUMEN
Este trabajo se realizó con dos propósitos: el primero es comparar los cambios manifestados en la estructura del empleo manufacturero durante el período de 1985-1988, en el municipio de Mexicali, en relación con el estado de Baja California y el país en su conjunto; para ello se hace uso de la técnica de cambio y participación (shift \& share) utilizandola información de los censos económicos. El segundo, relacionado con el anterior, se refiere a la aplicación de las tres versiones de dicha técnica a fin de determinar cuál de éstas presenta mayores elementos para la descripción de los cambios observados.

El trabajo muestra que la entidad presenta ventajas de localización en las actividades de manufactura de productos metálicos, maquinaria y equipos e instrumentos quirúrgicos y de precisión; mientras que en Mexicali se registran ventajas para las actividades de papel y productos de papel y productos minerales no metálicos, aun cuando no se reporta especialización.

Finalmente, se concluye que el modelo síntesis, presentado por Bishop y Simpson, es el que aporta mayores elementos para la descripción.
\end{abstract}

\begin{abstract}
The paper presented has two purposes: first, to compare the changes from 1985 to 1988 on the employment structure in the manufacturing industry in Mexicali compared with that of the state of Baja California and also with the country as a whole. For that matter a shift \& share model is used to analyze data from the economic census. The second purpose is related to the first one: refers to the application of three versions of the shift \& share technique, in order to estimate which one provides a more accurate description of the changes observed.

The paper demonstrates that Baja California has locational advantages in activities classified as non metallic manufacturing products and machinery, surgical and precision equipment and tools. Mexicali shows also advantages for activities in the paper product industry as well as in non metallic mineral products, even though there is no evidence of specialization.

Finally, it is concluded that the Bishop \& Simpson synthesis model provides more elements for an accurate description.
\end{abstract}

* Estudiantes de la Maestría en Ciencias Sociales Aplicadas. Opción Desarrollo Urbano y Regional. IS-UABC. 


\section{INTRODUCCIÓN}

La crisis económica sufrida en México, principalmente durante los primeros seis años de la década pasada, se reflejó en un crecimiento negativo del PIB, que pasó de una tasa de crecimiento del $7.9 \%$ en 1981 al $-3.9 \%$ en 1986 (Tello, 1989: 10, cuadro 1).

Por su parte, el empleo registró tasas de crecimiento negativas durante 1982 y 1983 (-0.9 y -1.5\% respectivamente) con una recuperación en 1984 (2.7\%). De esta caída en el empleo, dos son los sectores que presentan las tasas de crecimiento negativo más altas en los años mencionados: construcción $(-5.1,-20.4$ y 3.3) y manufactura $(-2.2$, -7.1 y 2.2 ) (véase: Tello, 1989: 438, cuadro b.3.5). Sin embargo, a pesar del crecimiento positivo del empleo durante 1984, dos años después el país de nuevo se ve inmerso en problemas financieros derivados del aumento de la deuda con el consecuente descenso de la actividad económica.

La crisis económica afectó de manera diferente a los diversos sectores productivos y también tuvo repercusiones diferenciales en las entidades federativas que conforman al país.

Un objetivo de este trabajo es profundizar en la descripción de los cambios en la estructura del empleo durante el período posterior a 1984, básicamente en el sector manufacturero. Para efecto de la comparación de los cambios registrados en el empleo del municipio de Mexicali, en relación con la entidad y con el país, se presenta la información desagregada de este sector correspondiente a 1985 y 1988, utilizando para ello los datos proporcionados por los censos económicos de 1986 y 1989.

Para el análisis de los datos, además de los números absolutos y relativos, se utiliza la técnica de cambio y participación (shift \& share), que permite estudiar de manera desagregada la dinámica de la estructura del empleo de una región en comparación con un nivel de agregación mayor. En este sentido, un segundo objetivo de este documento es comparar los resultados obtenidos de la aplicación de los tres enfoques de esta técnica (versión americana, británica y la propuesta por Bishop y Simpson) para determinar cuál describe mejor los cambios en la estructura del empleo.

El trabajo se enfoca principalmente al sector manufacturero, dado que a nivel nacional fue uno de los más afectados por la crisis económica y, por otra parte, debido a que la información disponible permite estudiar la estructura interna de este sector a nivel desagregado. 


\section{POBLACIÓN Y EMPLEO}

La descripción de la distribución y crecimiento del empleo por sectores permite un acercamiento inicial al conocimiento de la estructura y el comportamiento de la economía de una región; comparar la velocidad del crecimiento de la población con el ritmo de crecimiento del empleo proporciona una noción de la adecuación del comportamiento de la economía a las necesidades de la sociedad en general.

En el cuadro 1 se observa que la población total del país en el periodo 1985-1988 creció en un 8.14\%, mientras que la población ocupada en el sector manufacturero decreció en un 4.08\%; situaciones diferentes se observan tanto para el estado de Baja California como para Mexicali, pues en estos últimos se registran números positivos, además el crecimiento del empleo manufacturero supera al de la población -más del doble en ambos casos-, que es indicador del dinamismo del sector tanto en la entidad como en el municipio, que, a pesar del decrecimiento nacional, logran crecer en forma importante. En síntesis, el crecimiento del empleo manufacturero en el estado y en el municipio es superior al registrado por las poblaciones respectivas, situación que en el país no se presenta. Ello se comprueba con los índices de cambio que muestran que la población de la entidad creció alrededor del $25 \%$ por arriba del país, sin embargo, el empleo en el sector manufacturero no sólo no decreció sino que aumentó en casi 8 veces el valor absoluto de la tasa nacional.

\section{ESTRUCTURA DEL EMPLEO EN EL SECTOR MANUFACTURERO}

De acuerdo con la información de los censos económicos de 1986 y 1989 referente al personal ocupado (ver cuadro 2), se observa que el estado de Baja California aumenta en forma importante su peso relativo en el empleo manufacturero nacional, pues pasa del 2 al $3 \%$. Es conveniente precisar que durante el periodo revisado el empleo manufacturero a nivel nacional decreció tanto en términos absolutos como relativos, pasando de 2'577,959 a 2'472,826; mientras que Baja California muestra un incremento porcentual del $35 \%$ del personal ocupado en el sector manufacturero entre 1985 y 1988, pasando de 56,707 a 76,691 empleados.

En México, se observa que, para 1985 y 1988, aproximadamente el $80 \%$ del total del empleo en el sector manufacturero se concentra en cuatro subsectores: productos alimenticios, bebidas y tabaco (31); textiles, prendas de vestir e industria del cuero (32); sustancias químicas, productos derivados del petróleo y del carbón, de hule y de plástico (35); y productos metálicos, maquinaria y equipos, instrumentos quirúrgicos y de precisión (38). De ellos, los de mayor importancia relativa en 1988 son: el subsector 38 (29\%) y el 31 (21\%). 
CUADRO 1. Comparación de la población total y empleo manufacturero 1985 y 1988.

\begin{tabular}{|c|c|c|c|c|c|c|c|c|}
\hline \multirow[b]{2}{*}{ Localidad } & \multicolumn{2}{|c|}{$\begin{array}{l}\text { Proyecciones para la } \\
\text { población total }\end{array}$} & \multicolumn{2}{|c|}{$\begin{array}{l}\text { Personal ocupado en } \\
\text { sector manufacturero }\end{array}$} & \multicolumn{2}{|c|}{$\begin{array}{c}\% \text { de la población } \\
\text { total }\end{array}$} & \multicolumn{2}{|c|}{$\begin{array}{c}\text { Incr. porcentual } \\
1985-1988\end{array}$} \\
\hline & 1985 & 1988 & 1985 & 1988 & 1985 & 1988 & $\begin{array}{c}\text { Población } \\
\text { total }\end{array}$ & $\begin{array}{l}\text { Pers. ocupado } \\
\text { en manufact. }\end{array}$ \\
\hline México & 71315621 & 77119947 & 2577959 & 2472826 & 3.61 & 3.21 & 8.14 & -4.08 \\
\hline Baja California & 1413127 & 1556938 & 56707 & 76691 & 4.01 & 4.93 & 10.18 & 35.24 \\
\hline \multirow[t]{3}{*}{ Mpio. Mexicali } & 542222 & 577300 & 20609 & 23385 & 3.80 & 4.05 & 6.47 & 13.47 \\
\hline & \multicolumn{2}{|c|}{ Índices de cambio } & & & & & & \\
\hline & Pob. total & P.oc. manuf. & & & & & & \\
\hline B.Cfa./México & 1.25 & 8.64 & & & & & & \\
\hline Mexicali/B. Cfa. & 0.64 & 0.38 & & & & & & \\
\hline Mexicali/México & 0.79 & 3.30 & & & & & & \\
\hline
\end{tabular}

FUENTE: Resultados de los censos económicos 1986 y 1989; Sistema automatizado de información censal, INEGi, México, 1991. $x$ censo general de población y vivienda. 1983, INEGI-SPP. XI censo general de población y vivienda, 1991, INEGI.

Las proyecciones se efectuaron a partir de la tasa de crecimiento del periodo 1970-1990 obtenida con la fórmula: $\mathrm{TC}=(\mathrm{Pf} / \mathrm{Pi})^{1 / n}-1 \times 100$ aplicada a los datos de los censos de población.

fndice de cambio $=(\mathrm{Pf}-\mathrm{Pi} / \mathrm{Pi}) \times 100$. 
En el estado se registran tres subsectores que, en conjunto, aglutinan más del 75\% del empleo en ambas fechas; para 1985 éstos fueron el 31, 32 y 38 ; mientras que en 1988 , el subsector $32(9 \%)$ es reemplazado en importancia por el $33(10 \%)$-que corresponde a la industria y productos de madera-manteniéndose la importancia de los dos restantes. De ellos, el de mayor peso relativo en ambas fechas es el 38 con casi el 50\% del empleo en 1988, seguido en importancia por el subsector 31, mismo que pierde peso relativo entre 1985 (23\%) y $1988(16 \%)$.

En cuanto a las diferencias más importantes en la distribución del empleo a nivel nacional y estatal, en el cuadro 3 se observa que:

a) En el estado, el subsector 38 -que corresponde a productos metálicos, maquinaria y equipos e instrumentos quirúrgicos y de precisiónconcentró en 1988 el $49 \%$ del empleo manufacturero, e incrementó su importancia relativa en 4 puntos porcentuales respecto a 1985 , mientras que para el país, si bien es el subsector más importante, su peso relativo es inferior al del estado en 16 puntos porcentuales, manteniéndose constante en ambas fechas y concentrando al $29 \%$ del empleo, lo que podría considerarse como un primer indicador de la especialización de la entidad en este tipo de actividades industriales.

b) Respecto al subsector 31, a nivel nacional mantiene su participación de aproximadamente $20 \%$, mientras que en el estado decrece su importancia al pasar del 23\% al 16\%; sin embargo, al igual que en el país, las actividades consideradas en dicho subsector: productos alimienticios, bebidas y tabaco, ocupan el segundo lugar en importancia en el sector.

c) El subsector 33 (industrias y productos de madera) mantiene su participación a nivel nacional, mientras que en el estado aumenta la proporción del empleo en el subsector pasando del 6 al $10 \%$ entre 1985 y 1988; por otro lado, en el estado ocupa el tercer lugar en orden de importancia, mientras que en el país le corresponde el sexto lugar. Estas últimas cifras sugieren una posible especialización en la entidad.

Respecto al municipio de Mexicali se observa que son tres los subsectores de actividad que concentran más del $80 \%$ del personal ocupado en ambas fechas, a saber: 31,32 y 38 . De ellos, el de mayor importancia, tanto en términos absolutos como en relativos, es el subsector 38, pues aumenta del 47 al 55\%, lo que es indicador de que la especialización que presenta el estado en este subsector puede obedecer a la participación del municipio de Mexicali. La otras dos actividades en orden de importancia son el 32 (14 y $15 \%$ respectivamente) y el 31 que disminuye drásticamente al pasar del $21 \%$ en 1985 al $11 \%$ del personal ocupado en 1988. 
CUADRO 2. Estructura del empleo en el sector manufacturero, México, Baja California, Mexicali, 1985 y 1988.

\begin{tabular}{|c|c|c|c|c|c|}
\hline \multirow{2}{*}{$\begin{array}{l}\text { Subsector } \\
\text { actividad } \\
\end{array}$} & \multirow[b]{2}{*}{1985} & \multicolumn{3}{|c|}{ Personal ocupado } & \multirow{2}{*}{$\begin{array}{c}\text { Tasa de cre } \\
85-88 \\
(1)\end{array}$} \\
\hline & & 1988 & $\begin{array}{c}1985 \\
\%\end{array}$ & $\begin{array}{c}1988 \\
\%\end{array}$ & \\
\hline \multicolumn{6}{|l|}{ México } \\
\hline 31 Productos alimenticios, bebidas y tabaco. & 524693 & 508596 & 20.35 & 20.57 & -0.03 \\
\hline 32 Textiles, prendas de vestir e industria del cuero. & 391490 & 413706 & 15.19 & 16.73 & 0.06 \\
\hline 33 Industrias de la madera y productos de madera. Incluye muebles. & 124441 & 127400 & 4.83 & 5.15 & 0.02 \\
\hline 34 Papel y productos de papel, imprentas y editoriales. & 133272 & 129960 & 5.17 & 5.26 & -0.02 \\
\hline $\begin{array}{l}35 \text { Sustancias químicas, productos derivados del petróleo y } \\
\text { del carbón, de hule y de plástico. }\end{array}$ & 375155 & 320455 & 14.55 & 12.96 & -0.15 \\
\hline $\begin{array}{l}36 \text { Productos minerales no metálicos. Excluye los derivados } \\
\text { del petróleo y del carbón }\end{array}$ & 142316 & 136281 & 5.52 & 5.51 & -0.04 \\
\hline 37 Industrias metálicas básicas. & 122790 & 91904 & 4.76 & 3.72 & -0.25 \\
\hline $\begin{array}{l}38 \text { Productos metálicos, maquinaria y equipos. Incluye instrumentos } \\
\text { quirúrgicos y de precisión. }\end{array}$ & 738148 & 715195 & 28.63 & 28.92 & -0.03 \\
\hline 39 Otras industrias manufactureras. & 25654 & 29329 & 1.00 & 1.19 & 0.14 \\
\hline Total & 2577959 & 2472826 & 100.00 & 100.00 & -0.04 \\
\hline \multicolumn{6}{|l|}{ Baja California } \\
\hline 31 Productos alimenticios, bebidas y tabaco. & 12852 & 12477 & 22.66 & 16.27 & -0.03 \\
\hline 32 Textiles, prendas de vestir e industria del cuero. & 5388 & 7185 & 9.50 & 9.37 & 0.33 \\
\hline 33 Industrias de la madera y productos de madera. Incluye muebles. & 3317 & 7737 & 5.85 & 10.09 & 1.33 \\
\hline 34 Papel y productos de papel, imprentas y editoriales. & 3276 & 3818 & 5.78 & 4.98 & 0.17 \\
\hline $\begin{array}{l}35 \text { Sustancias químicas, productos derivados del petróleo y } \\
\text { del carbón, de hule y de plástico. }\end{array}$ & 2548 & 4066 & 4.49 & 5.30 & 0.60 \\
\hline
\end{tabular}


36 Productos minerales no metálicos. Excluye los derivados del petróleo y del carbón

37 Industrias metálicas básicas.

38 Productos metálicos, maquinaria y equipos. Incluye instrumentos quirúrgicos y de precisión.

39 Otras industrias manufactureras.

Total

\begin{tabular}{rrrrr}
1594 & 2797 & 2.81 & 3.65 & 0.75 \\
394 & 353 & 0.69 & 0.46 & -0.10 \\
25396 & 37516 & 44.78 & 48.92 & 0.48 \\
& & & & \\
1988 & 742 & 3.51 & 0.97 & -0.63 \\
56753 & 76691 & 100.00 & 100.00 & 0.35 \\
& & & & \\
4364 & 2490 & 21.18 & 10.65 & -0.43 \\
2991 & 3333 & 14.51 & 14.25 & 0.11 \\
400 & 631 & 1.94 & 2.70 & 0.58 \\
992 & 1542 & 4.81 & 6.59 & 0.55 \\
779 & 1160 & 3.78 & 4.96 & 0.49 \\
& & & & \\
476 & 1017 & 2.31 & 4.35 & 1.14 \\
& & & & \\
208 & 26 & 1.01 & 0.11 & -0.88 \\
9695 & 12897 & 47.04 & 55.15 & 0.33 \\
704 & 289 & 3.42 & 1.24 & -0.59 \\
20609 & 23385 & 100.00 & 100.00 & 0.13 \\
\hline
\end{tabular}

Mexicali

31 Productos alimenticios, bebidas y tabaco.

32 Textiles, prendas de vestir e industria del cuero.

33 Industrias de la madera y productos de madera. Incluye muebles.

34 Papel y productos de papel, imprentas y editoriales.

35 Sustancias químicas, productos derivados del petróleo y del carbón, de hule y de plástico.

36 Productos minerales no metálicos. Excluye los derivados del petróleo y del carbón

37 Industrias metálicas básicas.

38 Productos metálicos, maquinaria y equipos. Incluye instrumentos quirúrgicos y de precisión.

39 Otras industrias manufactureras.

Total

FUENTE: Resultados de los censos económicos 1986 y 1989, Sistema Automatizado de Información Censal, INEGI, México, 1991.

Notas: (1) Porcentaje de crecimiento en el periodo $=(\mathrm{E} 1-\mathrm{Eo}) / \mathrm{Eo}$, donde $\mathrm{E} 1$ = empleo final, $\mathrm{E}$ o $=$ empleo inicial. 
Como puede apreciarse en el cuadro 3, mientras que en 1988 el estado participa con el $3.1 \%$ del empleo manufacturero del país, los subsectores 33 y 38 destacan tanto por su aumento porcentual como por su fuerte peso relativo; por otra parte, se observa una importante disminución de la participación del subsector 39. Para el caso de Mexicali, en 1988 este subsector participa con el $0.95 \%$ del empleo manufacturero del país lo que representa un incremento del $.15 \%$ en el periodo, y en relación con la entidad representa alrededor del $30 \%$. En comparación con el país sólo dos subsectores $(34$ y 38 ) registran un peso superior al del conjunto del municipio, lo cual indica una posible especialización en ellos. En relación con la entidad se observa una disminución de la participación del municipio en el empleo manufacturero durante el periodo estudiado, especialmente en el subsector 37, aumentando la participación relativa de los subsectores 34,36 y 39 y destacan, por su importancia, las actividades relacionadas con los subsectores 32,34 y 39 .

Para identificar con mayor claridad la concentración de actividades industriales, tanto en el estado como en el municipio, en el cuadro 4 se presentan los cocientes de localización, donde se observa que en 1988 Baja California concentra actividades en los subsectores 33 y 38 . Por su parte, Mexicali, en el contexto del estado, muestra concentración de actividades

\section{CUADRO 3. Baja California y Mexicali. Comparación de porcen- tajes de participación del empleo manufacturero por subsector, 1985 y 1988.}

\begin{tabular}{rcccrcc}
\hline & \multicolumn{2}{c}{ B.C./México } & \multicolumn{2}{c}{ Mexicali/B.C. } & \multicolumn{2}{c}{ Méxicali/México } \\
Subsector & 1985 & 1988 & 1985 & 1988 & 1985 & 1988 \\
\hline 31 & 2.45 & 2.45 & 33.96 & 19.96 & 0.83 & 0.49 \\
32 & 1.38 & 1.74 & 55.51 & 46.39 & 0.76 & 0.81 \\
33 & 2.67 & 6.07 & 12.06 & 8.16 & 0.32 & 0.50 \\
34 & 2.46 & 2.94 & 30.28 & 40.39 & 0.74 & 1.19 \\
35 & 0.68 & 1.27 & 30.57 & 28.53 & 0.21 & 0.36 \\
36 & 1.12 & 2.05 & 29.86 & 36.36 & 0.33 & 0.75 \\
37 & 0.32 & 0.38 & 52.79 & 7.37 & 0.17 & 0.03 \\
38 & 3.44 & 5.25 & 38.18 & 34.38 & 1.31 & 1.80 \\
39 & 7.75 & 2.53 & 35.41 & 38.95 & 2.74 & 0.99 \\
Total & 2.20 & 3.10 & 36.31 & 30.49 & 0.80 & 0.95 \\
\hline
\end{tabular}

FUENTE: Cálculos efectuados a partir del cuadro 2. 
en los subsectores $32,34,36,38$ y 39 ; sin embargo, la aparente especialización en el subsector 39 deriva de que su peso relativo en el estado es ligeramente inferior al del municipio aunque muestra tasas negativas de crecimiento en ambos casos, no obstante, en el estado decreció más pronunciadamente que en el municipio.

En relación con el país, Mexicali concentra actividades en los subsectores 34 y 38 , y resulta interesante destacar que el municipio muestra una concentración de las actividades en ellos consideradas tanto en comparación con el estado como con el país. Cabe señalar que la concentración de actividades en el subsector 33 a nivel estatal probablemente no corresponda a Mexicali sino a algún otro municipio de la entidad.

Finalmente, la concentración de actividades en los subsectores 34 y 38 en Mexicali se confirma tanto a través de las tasas de crecimiento y la participación relativa, como con los cocientes de localización.

CUADRO 4. Cocientes de localización para el sector manufacturero. Baja California y Mexicali, 1985 y 1988

\begin{tabular}{rcccccc}
\hline & \multicolumn{2}{c}{ B.C.-México } & \multicolumn{2}{c}{ Mxli.-B.C. } & \multicolumn{2}{c}{ Mxli.-México } \\
Subsector & 1985 & 1988 & 1985 & 1988 & 1985 & 1988 \\
\hline 31 & 1.11 & 0.79 & 0.93 & 0.65 & 1.04 & 0.52 \\
32 & 0.63 & 0.56 & 1.53 & 1.52 & 0.96 & 0.85 \\
33 & 1.21 & 1.96 & 0.33 & 0.27 & 0.40 & 0.52 \\
34 & 1.12 & 0.95 & 0.83 & 1.32 & 0.93 & 1.25 \\
35 & 0.31 & 0.41 & 0.84 & 0.94 & 0.26 & 0.38 \\
36 & 0.51 & 0.66 & 0.82 & 1.19 & 0.42 & 0.79 \\
37 & 0.15 & 0.12 & 1.45 & 0.24 & 0.21 & 0.03 \\
38 & 1.56 & 1.69 & 1.05 & 1.13 & 1.64 & 1.91 \\
39 & 3.52 & 0.82 & 0.97 & 1.28 & 3.43 & 1.04 \\
Total & 1.00 & 1.00 & 1.00 & 1.00 & 1.00 & 1.00 \\
\hline
\end{tabular}

FUENTE: Cálculos realizados a partir de información de los censos económicos de 1986 y 1989.

Fórmula : $\mathrm{CL}=(\mathrm{Eir} / \mathrm{Er}) /(\mathrm{Ein} / \mathrm{En})$

Donde: $\mathrm{Eir}=$ empleo en la industria i en la región.

$\mathrm{Er}=$ empleo total industrial (sector) en la región.

Ein $=$ empleo en la industria $i$ en la nación.

En = Empleo total industrial en la nación. 


\section{TÉCNICA DE CAMBIO Y PARTICIPACIÓN}

La técnica de los componentes del cambio (shift \& share) se utiliza para comparar cl crecimiento, ya sea del empleo o del valor del producto, de una región contra un nivel de agregación mayor -que gencralmente es el nacional-. Supone que el comportamiento de la región en estudio debería mostrar el mismo ritmo de crecimiento que el de su referente, a menos que presente ventajas o desventajas comparativas.

Existen diversas versiones de la técnica, de éstas Bishop y Simpson (1972) indican que dos son las más utilizadas: la versión británica y la americana. Según estos autores, ambas presentan limitaciones al interpretar los comportamientos de las industrias particulares, aunque a nivel agregado los resultados sean iguales; por ello, presentan una propuesta de síntesis que intenta reducir las deficiencias de las otras versiones.

Esta técnica parte de la descomposición del crecimiento del empleo regional en dos componentes: "el crecimiento que hubiese ocurrido si las industrias en la región hubieran crecido a las tasas nacionales; y un residual, o componente diferencial, que representa los efectos de las variaciones entre las tasas de crecimiento de la industria regional y nacional" (Bishop y Simpson, 1972:60). El primer elemento, o crecimiento potencial, a su vez se desdobla en dos componentes: el nacional y el estructural.

El componente diferencial muestra si el crecimiento del empleo en las ramas industriales, o el total industrial, en la región es más rápido o lento que el de su referente, es decir, "permite analizar el grado de motricidad que alcanzan éstas durante las fases estudiadas" e indica la posición competitiva (Sández Pérez, 1987: 74).

El componente estructural refleja la composición industrial, permitc identificar las industrias básicas y, además, cuando el resultado es "positivo y grande en algún sector, indica que la región encuentra ventajas locacionales en la especialización en esa actividad" (Sández. Pérez, 1987: 74).

La notación utilizada para la aplicación de esta técnica es:.

$\mathrm{e}=$ Empleo de la industria $i$ en la región en el año base.

$\mathrm{E}=$ Empleo total industrial en la región en el año base.

$\mathrm{n} \quad=$ Empleo de la industria $i$ en la nación en el año base.

$\mathrm{N}=$ Empleo total industrial en la nación en el año base.

$\mathrm{e}^{\mathbf{\prime}}=$ Tasa de crecimiento del empleo en la industria $i$ regional.

$\mathrm{E}^{\prime}=$ Tasa de crecimiento del empleo total industrial regional.

$\mathrm{n}^{\prime}=$ Tasa de crecimiento del empleo de la industria $i$ nacional.

$\mathrm{N}^{\prime}=$ Tasa de crecimiento del empleo total industrial nacional. 
Los autores que han usado esta técnica (Bishop y Simpsom, 1972; Unikel, et al., 1976; Sández Pérez, 1987; Castillo, 1990) advierten que por sí sola no explica el crecimiento económico de una región, es decir, que carece de sustento teórico. Por otra parte, puede conducir a proyecciones excesivamente optimistas o encubrir los efectos de factores cconómicos internos y/o externos que operan en la región.

Cabe aclarar que además de las deficiencias señaladas, los resultados obtenidos pueden variar según sea la versión de la técnica utilizada, el tipo de estandarización empleado, el nivel de agregación, el período estudiado $y$ los problemas de compatibilidad de información.

Con el propósito de comparar la aplicación de las tres versiones presentadas por Bishop y Simpson (1972), se muestran los resultados de los componentes diferencial (cuadro 5), estructural y nacional (cuadro 6) obtenidos para el municipio de Mexicali y el estado de Baja California para los años de 1985 y 1988, correspondientes a la estructura de empleo del sector manufacturero.

El componente diferencial refleja el empleo final del periodo en la región si éste hubiera crecido o decrecido de acuerdo con la diferencia entre las tasas de crecimiento de la industria en la región y la nación -0 nivel de agregación mayor-. Es decir, muestra el saldo, positivo o negativo, de los empleos que presenta la región observada. Por otro lado, el componente diferencial es indicador de la posición de ventaja o desventaja competitiva.

El cálculo de este componente es el mismo en las tres versiones de la técnica, y los resultados presentados en el cuadro 5 indican que, a nivel agregado, la entidad presenta ventaja en relación con el país, ya que se registra un total de 21,073 empleos "excedentes", que se explican por el hecho de que todos los subsectores, salvo el 39 , registran números positivos al compararlos con el país. De ellos destaca el subsector 38 y, en menor medida, los subsectores $33,35,32$ y 36 , que en conjunto son los que presentan ventajas competitivas en la entidad respecto del país.

La situación observada en el caso del municipio, al ser comparado con la entidad, es opuesta a la descrita en el párrafo anterior. Mexicali no creció al ritmo de la entidad, de aquí que se registre un "faltante" de 3,779 empleos y sólo presente un crecimiento mayor que la entidad en los subsectores 34,36 y 39 , aunque los números son bajos cuando se comparan con las magnitudes de las cifras negativas. No obstante, al compararse con el país el saldo global es positivo, confirmándose la pérdida de dinamismo en los subsectores 31 y 37 tanto en relación a la entidad como con el país. De esta manera, se observa que el municipio pierde dinamismo respecto a la entidad pero aun así es superior al estándar nacional. 
Por otra parte, el hecho de que los resultados de los subsectores 33 y 35 sean positivos en Mexicali en relación con el país, pudiera ser indicador de ventajas, no obstante éstas se desvanecen al compararse con la entidad. Por ello, los únicos subsectores que pudieran presentar una posición de ventaja real en el municipio son los subsectores 34 y 36 aun cuando no se reporte especialización en ellos .

Por otra parte, el componente nacional (ver cuadro 6), que refleja el crecimiento de las industrias si éstas se hubieran comportado siguiendo el estándar nacional, muestra variaciones importantes, sobre todo en cuanto al signo en las tres versiones. Para el caso del estado de Baja California en relación al país, aparece un "excedente" de 2,315 trabajadores, mientras que para el municipio es de 840 . Sin embargo, al contrastar el municipio con el estado, se observa un "faltante" de 7,240 empleos debido a que el dinamismo de la entidad es mucho mayor que el del municipio.

En relación con el componente estructural (cuadro 6), se abvierte que los resultados totales en las tres versiones son idénticos, las diferencias surgen a nivel de subsector, en algunos casos no sólo en signo sino también en magnitud. De esta manera, a nivel agregado, se observa que Baja California en comparación con el país presenta "ventajas de especialización" en el sector manufacturero; lo mismo puede decirse del municipio de Mexicali en relación con la nación, aun cuando la magnitud es inferior. Por otra parte, Mexicali no presenta una ventaja de especialización del sector manufacturero en el contexto del estado, situación que indica que las mayores ventajas, en términos generales, no se localizan en Mexicali.

$\mathrm{Al}$ estudiar los resultados del componente estructural correspondiente a Baja California en su relación con México puede apreciarse que las versiones británica y Bishop-Simpson coinciden en signo y jerarquía en los subsectores con mayor magnitud en la entidad, a saber: 35,37 y 39 ; mientras que la versión americana discrepa con las anteriores tanto en signo como en jerarquía.

Cabe señalar que el resultado positivo para el componente estructural en el subsector 39 (que indica "ventaja de especialización") discrepa con el signo negativo y la magnitud del componente diferencial que indica una importante pérdida de dinamismo relativo en ese subsector. Esto podría tener como explicación que al estar perdiendo empleos y por tanto la especialización, la entidad registra ventajas derivadas de un grupo de actividades que a nivel nacional no son las más dinámicas; y por otra parte, esto puede ser resultado de la gran diversidad de actividades incluidas en este subsector. ${ }^{1}$ Por otro lado, aunque hay un crecimiento importante en los subsectores 32 y 33 , los resultados del componente estructural no indican especialización en ellos. 
CUADRO 5. Componente diferencial para las tres versiones, Baja California y Mexicali, 1985-1988.

\begin{tabular}{lrrr}
\hline Subsector & $\begin{array}{c}\text { B.C./México } \\
\text { e(e'-n') }\end{array}$ & $\begin{array}{c}\text { Mexicali/B.C. } \\
\text { e(c'-n') }\end{array}$ & $\begin{array}{c}\text { Mexicali/Mexico } \\
\text { e(e'-n') }\end{array}$ \\
\hline 31 & 19.29 & -1746.67 & -1740.12 \\
32 & 1491.25 & -655.56 & 172.27 \\
33 & 4341.13 & -302.01 & 221.49 \\
34 & 623.41 & 385.88 & 574.65 \\
35 & 1889.51 & -83.10 & 494.58 \\
36 & 1279.59 & 181.76 & 561.19 \\
37 & 58.10 & -160.36 & -129.68 \\
38 & 12909.70 & -1424.85 & 3503.47 \\
39 & -1530.79 & 26.24 & -515.85 \\
Total & 21072.20 & -3778.66 & 3142.00 \\
\hline
\end{tabular}

FUENTE: Cálculos realizados a partir de la información del cuadro 2, con las fórmulas que parecen en cada columna.

En la descripción presentada en la segunda parte de este trabajo (ver cuadros 2 a 4) se señalaron a los subsectores 33 y 38 como los más dinámicos y los que concentraban una mayor proporción del empleo al interior de la entidad; considerando esto, la versión Bishop-Simpson es la que mejor los representa.

Los resultados para el municipio en comparación con el país permiten observar la coincidencia en signo y jerarquía entre las versiones británica y Bishop-Simpson en la mayor parte de los subsectores. De acuerdo con estos resultados puede suponerse que hay ventaja de especialización en los subsectores $35,37,38$ y 39 . Respecto de este último se puede interpretar en el mismo sentido que en el caso de Baja California con relación al país. Inicialmente se identificaron los subsectores 34 y 38 como aquellos en los que el municipio mostraba una importante concentración del empleo y que además se encontraban entre los más dinámicos; en este sentido, y considerando las cifras de los componentes estructural y diferencial, la versión que mejor describe esta situación es la Bishop-Simpson.

1 Actividades incluidas en el subsector 39: fabricación de joyas y orfebrería, acuñación de monedas, fabricación de instrumentos musicales, de artículos y útiles de oficina y dibujo, de juguetes, de escobas y cepillos, de joyas de fantasía, sellos metálicos y de gorna, cierres de cremallera, otros productos no clasificados en otra parte y fabricación y reparación de aparatos e instrumentos para pesar (NNGI, 1988:119). 
CUADRO 6. Resultados de los componentes nacional y estructural en las tres versiones.

\begin{tabular}{|c|c|c|c|}
\hline Subsector & $\begin{array}{l}\text { Versión americana } \\
\text { Componente }\end{array}$ & $\begin{array}{l}\text { Versión británica } \\
\text { Componente }\end{array}$ & $\begin{array}{l}\text { Síntesis Bishop-Simpson } \\
\text { Componente }\end{array}$ \\
\hline de actividad & Nacional Estructural & Nacional Estructural & Nacional Estructural \\
\hline
\end{tabular}

\begin{tabular}{|c|c|c|c|c|c|c|}
\hline \multicolumn{7}{|c|}{ Baja California en relación con el país } \\
\hline 31 & -524.12 & 129.84 & -354.37 & -39.91 & -407.43 & 13.14 \\
\hline 32 & -219.73 & 525.49 & 489.08 & -183.32 & 620.82 & -315.07 \\
\hline 33 & -135.27 & 214.14 & 65.14 & 13.73 & 41.59 & 37.28 \\
\hline 34 & -133.60 & 52.19 & -72.91 & -8.50 & -86.86 & 5.45 \\
\hline 35 & -103.91 & -267.60 & -1204.20 & 832.69 & -971.30 & 599.79 \\
\hline 36 & -65.01 & -2.59 & -132.86 & 65.26 & -70.09 & 2.50 \\
\hline 37 & -16.07 & -83.04 & -679.95 & 580.84 & -585.77 & 486.67 \\
\hline 38 & -1035.69 & 245.99 & -505.30 & -284.40 & -878.29 & 88.59 \\
\hline 39 & -81.07 & 365.86 & 80.90 & 203.88 & 22.86 & 261.92 \\
\hline Total & -2314.47 & 1180.27 & -2314.47 & 1180.27 & -2314.47 & 1180.27 \\
\hline
\end{tabular}

Mexicali en relación con el estado

$\begin{array}{lrrrrrr}31 & 1533.12 & 1660.46 & -136.18 & 8.84 & -242.63 & 115.29 \\ 32 & 1050.77 & -53.22 & 652.55 & 345.00 & 1015.96 & -18.41 \\ 33 & 140.52 & 392.49 & 1605.06 & -1072.04 & 1322.42 & -789.41 \\ 34 & 348.50 & -184.38 & 196.82 & -32.70 & 127.39 & 36.73 \\ 35 & 273.67 & 190.43 & 551.24 & -87.14 & 499.85 & -35.76 \\ 36 & 167.22 & 192.02 & 436.85 & -77.61 & 400.72 & -41.48 \\ 37 & 73.07 & -94.72 & -14.89 & -6.76 & 7.92 & -29.56 \\ 38 & 3405.97 & 1220.88 & 4401.20 & 225.65 & 4567.30 & 59.54 \\ 39 & 247.32 & -688.56 & -452.47 & 11.23 & -458.76 & 17.52 \\ \text { Total } & 7240.19 & -685.53 & 7240.19 & -685.53 & 7240.19 & -685.53\end{array}$

Mexicali en relación con el país

\begin{tabular}{lrrrrrr}
31 & -177.97 & 44.09 & -128.68 & -5.20 & -135.59 & 1.71 \\
32 & -121.98 & 291.71 & 177.60 & -7.87 & 183.26 & -13.53 \\
33 & -16.31 & 25.82 & 23.66 & -14.14 & 47.91 & -38.40 \\
34 & -40.46 & 15.80 & -26.48 & 1.82 & -23.48 & -1.17 \\
35 & -31.77 & -81.81 & -437.29 & 323.71 & -346.75 & 233.17 \\
36 & -19.41 & -0.77 & -48.25 & 28.06 & -21.26 & 1.07 \\
37 & -8.48 & -43.84 & -246.91 & 194.59 & -215.36 & 163.04 \\
38 & -395.38 & 93.91 & -183.49 & -117.98 & -338.22 & 36.75 \\
39 & -28.71 & 129.56 & 29.38 & 71.47 & 9.03 & 91.82 \\
Total & -840.47 & 474.47 & -840.47 & 474.47 & -840.47 & 474.47 \\
\hline
\end{tabular}

FUENTE: Resultados de los Censos Económicos 1986 y 1989; Sistema Automatizado de Información Censal. INEGI, México, 1991.

Fórmulas:

Componente nal.

Comp. estructural versión americana

$\mathrm{eN}^{\prime}$

e $\left(n^{\prime}-N^{\prime}\right)$ ver. británica

$\mathrm{n}(\mathrm{E} / \mathrm{N}) \mathrm{n}^{\prime}$

$[e-(n E / N)] n^{\prime}$ ver. Bishop y Simpson $e N^{\prime}+n(E / N)\left(n^{\prime}-N^{\prime}\right)$ $\left[e-n(E / N)\left(n^{\prime}-N^{\prime}\right)\right.$ 
$\mathrm{Al}$ analizar los resultados correspondientes a Mexicali en su relación con Baja California la versión americana identifica a los subsectores 38 , 33,36 y 35 como los que muestran mayores "ventajas de especialización"; en la versión británica la jerarquía aparece de la siguiente forma: 32,38 , 39 y 31 ; mientras que en la versión Bishop-Simpson es 31, 38, 34 y 39. Por lo que Mexicali debiera atender las condiciones e infraestructura favorable a la industria alimentaria, la metal-mecánica, del papel e industrias diversas.

Es importante destacar que el periodo en estudio es particularmente difícil, debido a la inestabilidad económica que a nivel nacional se refleja en el decrecimiento del empleo en el sector manufacturero, tanto en el total como en la mayoría de los subsectores, mientras que el empleo manufacturero en la entidad y el municipio en términos generales crecen; lo anterior posiblemente influya en los resultados obtenidos dificultando su interpretación, particularmente si ésta se apoya en una elaboración analítica tan pobre como la versión americana. Por ello sería conveniente efectuar el ejercicio de comparación seleccionando un periodo de mayor estabilidad, o con crecimiento del empleo en los distintos niveles de agregación.

\section{OBSERVACIONES FINALES}

A manera de recapitulación, en principio destaca el hecho de que el empleo manufacturero en la entidad crece a un ritmo superior que la población (3 veces más rápidamente), mientras que para Mexicali la tasa de cambio durante el período analizado corresponde al doble de la correspondiente a la población. Ambas situaciones contrastan con el declinamiento del empleo manfacturero en el país.

En cuanto a la estructura del empleo en el estado de Baja California destacan por su importancia más que proporcional, en comparación con la nación, las actividades consideradas en el subsector 38 seguido por el 33; y por otra parte, aun cuando la industria alimenticia, tabaco y bebidas (31) segunda en importancia, aporta el $16.27 \%$ del empleo, muestra la misma tendencia de crecimiento negativo que la nacional, lo que refleja las dificultades de esta industria. Para el caso del municipio de Mexicali destacan por su importancia las actividades del subsector 38 que concentra más del $50 \%$ del empleo manufacturero.

La importancia de este tipo de actividades se confirma con los cocientes de localización, que muestran para el estado una concentración de actividades en los subsectores 33 y 38 . Para el municipio de Mexicali los valores registrados favorecen las actividades de los subsectores 34 y 38 . 
La información hasta aquí reseñada se confirma con la aplicación de la técnica de cambio y participación (shift \& share), que en términos generales indica un mayor dinamismo en la entidad en relación con México al mostrar un excedente de empleos, tanto en la entidad como en el municipio. Las ventajas de la localización son evidentes en Baja California para el subsector 38 y en menor medida para el 33, 35, 32 y 36 ; mientras que Mexicali registra ventajas en los subsectores 34 y 36 , aun cuando no se reporta especialización. El municipio aparece con especialización en el subsector 38, tanto en relación con el país como con el estado.

Finalmente, en cuanto a la determinanción del modelo que aporta mayores elementos para la descripción adecuada de la estructura y dinámica del empleo manufacturero, se puede afirmar que si bien las tres versiones de la técnica presentan inconsistencias a nivel de subsectores, es la síntesis propuesta por Bishop y Simpson (1972) la que presenta una mayor congruencia y estabilidad; además, es la versión que menos exagera las ventajas, debido a que, como puede observarse en las fórmulas aplicadas el modelo, estandariza doblemente, tanto en el aspecto estructural como en el de crecimiento. No obstante, es posible que el periodo analizado (sólo tres años), así como el declinamiento del empleo a nivel nacional y el nivel de agregación utilizado, sean elementos que influyan en los resultados obtenidos.

\section{BIBLIOGRAFÍA}

BISHOP, K. C. y C. E. Simpson 1972. "Components of Change Analysis: Problems of Alternative Approaches to Industrial Structure", Regional Studies. Vol 6, Pergamon Press, G.B.

CASTILLO, Víctor. 1990. Economí fronteriza y desarrollo regional. UABC, Mexicali.

INEGI 1987. Censos económicos 1986. Resultados oportunos estatales de Baja California. México, D.F.

- 1988. Clasificación mexicana de actividades y productos (CMAP). Aguascalientes, Ags.

- 1991. Censos económicos 1989. Resultados oportunos estatales de Baja California. Aguascalientes, Ags.

- 1991a. Sistema automatizado de información censal. Ags.

- 1991b. XI censo general de población y vivienda. Resultados definitivos. México.

INEGI-SPP. 1983. X censo general de población y vivienda. Para Baja California. México. 
SÁNDEZ Pérez, Agustín. 1987. "Estructura y dinámica del sector manufacturero bajacaliforniano (1960-1985)". Cuadernos de ciencias sociales, serie 3, no. 7. IIS-UABC, Mexicali.

TELLO, Carlos (coord.) 1989. México: Informe sobre la crisis (19821986). UNAM, México.

UNIKEL, et al. 1976. El desarrollo urbano de México. El Colegio de México, México, 2a. ed. 